\title{
Generation of 2D Conforming Voronoi Diagram in Complex Domain
}

\author{
Qi $\mathrm{Li}^{1}$, Haisheng $\mathrm{Li}^{1}$, Qiang $\mathrm{Cai}^{1}$, and Yuewu $\mathrm{Liu}^{2}$ \\ ${ }^{1}$ College of Computer and Information Engineering \\ Beijing Technology and Business University \\ Beijing, P.R. China, 100048 \\ ${ }^{2}$ Institute of Mechanics \\ Chinese Academy of Sciences \\ Beijing, P.R. China, 100190 \\ Qilidy@126.com
}

\begin{abstract}
The concept of two-dimensional conforming Voronoi diagram is given in this paper. The necessary and sufficient conditions on the existence of conforming conditions in the conforming Voronoi cells are analyzed and proved. A two-dimensional conforming Voronoi cells generation algorithm in complex domain is proposed. Example shows the effective of the proposed algorithm. The algorithm proposed in this paper could be widely used in various applications.
\end{abstract}

Keywords: Voronoi diagram, Delaunay triangulation, conforming, 2D complex domain.

\section{Introduction}

Oil reservoir numerical simulation has a very wide range of applications in the development of oil and gas field, which is able to effectively improve policy decision level of oil and gas field development. In oil reservoir numerical simulation, partial differential equation numerical method is usually used to solve equations. Therefore, discrete grid system used to partition the computational domains is an important factor influencing the stability of analog computation and the accuracy of computed result.

In oil reservoir numerical simulation field, Cartesian mesh was widely used first and played a tremendous role in the early days of analog computation. However, Cartesian mesh itself also has significant limitations, which include as below: (1) In some cases, Cartesian mesh has serious Grid Orientation problem. (2) Under complicated geological conditions, Cartesian mesh is not able to give a good description of oil reservoir morphology (such as boundary, fault). (3) Cartesian mesh is not able to represent the information of well position flexibly.

In oil reservoir numerical simulation field, conforming Voronoi diagram is called PEBI grid. PEBI (Perpendicular bisection) grid was proposed by Heinemann [1] in 1989. Melichar [2] pointed out, that using PEBI grid to describe geology and the 
information of well position is able to apply to the regular reservoir simulator based on structured Cartesian mesh or corner-point grid, and need not change workflow dramatically. Because PEBI grid is a unstructured locally orthogonal grid, it can give a good description of the boundary and fault of irregular geology, and is easy to make a local refinement. In addition, PEBI grid takes advantage of orthogonality of FDM (finite difference method), which makes it easy to transplant numerical simulation program [3] [4]. Consequently, PEBI grid has a good application prospect in oil reservoir numerical simulation field.

At present, researches on PEBI grid generation methods can never meet the practical requirements of the situation. Heinemann[1], Verma[5], Haibing Xie[3][4] proposed 2D PEBI grid generation methods, and these algorithms are simple and easy to implement. But conforming conditions of these algorithms are relatively simple and only contain outer boundary and well point, and they do not contain inner boundary. So these algorithms are hoped to be improved correspondingly to meet the current application requirements. KAPPA [6] used the method of scanning boundary points and discrete points in domain to generate smooth Delaunay triangulation grid first, and then generate PEBI grid. The method has a good effectiveness, but implementation of the algorithm is a little complex. So the algorithm is hoped to be improved suitably to make it easy to implement. Zuping Xiang [7] also proposed a generation method of 2D PEBI grid. The method assumes that inner boundary(such as fault) and outer boundary are straight-line segment, and takes mutual interference of nodes near the boundary, corner points and nodes near the vertical well into consideration. The algorithm is universal relatively, but because it need deal with about 10 interference circumstances, the algorithm is relatively complex. So the algorithm is hoped to be improved suitably to make it easier. Quanyi Yang [8], Qiang Cai[9] proposed control circle algorithms for generating PEBI grid, and the algorithms are simple, easy to implement and efficient. The algorithms can solve the generation problem of PEBI grid in ordinary conforming conditions, but its robustness is not very good and it is not convergent in some certain complex conforming conditions. So the algorithms are hoped to be improved properly to make them more robust. Jigang Li [10], Qin Yang [11] proposed Voronoi subdivision algorithms for generating 2D PEBI grid. The algorithms have good generality, and are able to deal with complex conforming conditions. However, the algorithms is easy to cause deformed grid cell, so the algorithms are hoped to be improved suitably to enable them to deal with all kinds of complex conforming conditions better.

On the basis of analyzing the necessary and sufficient conditions on the existence of conforming conditions in the 2D conforming Voronoi diagram, a conforming Voronoi diagram generation algorithm in 2D complex domain is proposed. The algorithm proposed in the paper has fast speed and wide adaptation, and the algorithm could be widely used in various applications.

\section{Basic Concepts of Conforming Voronoi Diagram}

Voronoi diagram is underlying data structure concerning space subdivision. The definition of Voronoi diagram is showed as below. 
Definition 1. Given $\boldsymbol{d}\left(\boldsymbol{x}, \boldsymbol{p}_{\boldsymbol{i}}\right)$ represents the Euclidean distance between point $\boldsymbol{x}$ and point $\boldsymbol{P}_{\boldsymbol{i}}$, neighborhood $\boldsymbol{V}_{\boldsymbol{i}}$ of point $\boldsymbol{P}_{\boldsymbol{i}}$ is defined as below:

$$
V_{i}=\left\{x \in R^{d} \mid d\left(x, p_{i}\right)<d\left(x, p_{j}\right), j \neq i\right\}
$$

Voronoi diagram is union of neighborhoods of all points in point set.

Connecting the points whose neighborhoods are adjacent in Voronoi diagram would generate Delaunay triangulation grid which is the dual graph of Voronoi diagram. As shown in Figure 1, every Voronoi vertex is the circumcentre of corresponding Delaunay triangle.

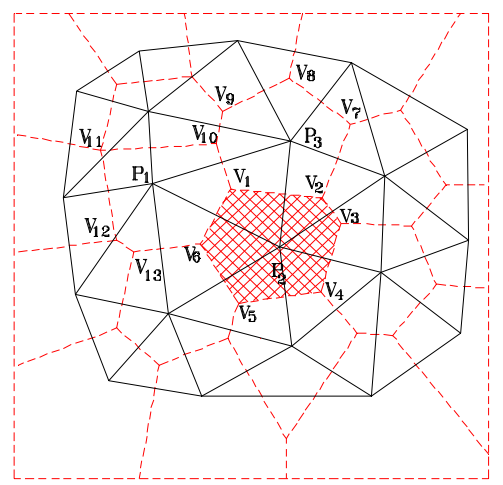

Fig. 1. Voronoi diagram and Delaunay triangulation grid

Conforming conditions of conforming Voronoi diagram possibly include:

(1) Conforming Point: the given points must be grid nodes.

(2) Conforming Line: the generated grids must distribute along meander line, and there must not be grids across conforming lines.

(3) Conforming Domain: there must not be grids inside inner conforming domain and outside outer conforming domain.

(4) Conforming Surface: a surface of grid block must be on given conforming surfaces, and there must not be grids across conforming surfaces.

As a general rule, conforming conditions are taken as Planar Straight Line Graph [12] (PSLG for short) in numerical grid generation field. Then PSLG is takes as the inputs of grid generation algorithms. PSLG $\boldsymbol{A}$ is a finite set of vertexes and segments, and fulfills the following two requirements: (1) Two end points of an arbitrary segment in Set $\boldsymbol{A}$ must be elements of Set $\boldsymbol{A}$. (2) Two arbitrary elements in Set $\boldsymbol{A}$, either do not intersect, or intersect at a public vertex. A PSLG $\boldsymbol{A}$ concerning oil reservoir area could always be got through normalization algorithm [13]. Assuming after normalizing, PSLG $\boldsymbol{A}$ are represented as union of a point set $\boldsymbol{P S}$ and a segment set $S S$, to wit $A=P S \bigcup S S$. 


\section{Conditions on the Existence of Conforming Conditions in Conforming Voronoi Diagram}

Generation of 2D conforming Voronoi diagram is to find a point set $\boldsymbol{S}_{\boldsymbol{V}}$ on the basis of given conforming condition PSLG $\boldsymbol{A}$, which fulfills the two requirements as showed below: (1)An arbitrary segment in $\boldsymbol{A}$ is union of some edges of conforming Voronoi diagram of $S_{V}\left(\right.$ denoted by $\left.\operatorname{CVD}\left(S_{V}\right)\right)$. (2)An arbitrary isolated point in $\boldsymbol{A}$ is a node of $\operatorname{CVD}\left(S_{V}\right)$.

Now, the key to the problem is how to find a growing point set $S_{V}$ to make $\operatorname{CVD}\left(S_{V}\right)$ fulfill conforming condition PSLG $\boldsymbol{A}$. Because an arbitrary isolated point in $\boldsymbol{A}$ must be a node of $\operatorname{CVD}\left(\boldsymbol{S}_{V}\right)$, all isolated points in $\boldsymbol{A}$ just need to be added to growing point set $S_{V}$. Then the next problem is what requirements $S_{V}$ should fulfill, so that make given segment $P Q$ in $\boldsymbol{A}$ become union of some edges of $\operatorname{CVD}\left(\boldsymbol{S}_{V}\right)$. The following two theorems will answer the question.

Theorem 1. The necessary and sufficient condition of that point $\boldsymbol{P}$ in PSLG $\boldsymbol{A}$ is a vertex of $\operatorname{CVD}\left(S_{V}\right)$ is: There exists a circle centered at $\boldsymbol{P}$, and the circle passes through at least three points in $S_{V}$ while no point in $S_{V}$ inside it.

Theorem 2. The necessary and sufficient condition of that segment $\boldsymbol{P} \boldsymbol{Q}$ in PSLG $\boldsymbol{A}$ is a edge of $\operatorname{CVD}\left(S_{V}\right)$ is: There exists two intersectant circles(intersection points denoted by $\boldsymbol{P}_{\boldsymbol{1}}$ and $\boldsymbol{P}_{2}$, and $P_{1} \in S_{V}, P_{2} \in S_{V}$ ) centered at $\boldsymbol{P}$ and $\boldsymbol{Q}$ respectively, and for each of those two circles there is at least one another point in $\boldsymbol{S}_{V}$ other than $\boldsymbol{P}_{\boldsymbol{l}}, \boldsymbol{P}_{\mathbf{2}}$ on it while no point in $S_{V}$ inside it.

On the basis of the concept of Voronoi diagram, theorem 1 is easy to be proved, and is omitted here. Theorem 2 will be proved here.

Proof. As shown in Figure 2, there exists circle $\boldsymbol{O}_{\boldsymbol{I}}$ centered at endpoint $\boldsymbol{P}$, and $\boldsymbol{O}_{\boldsymbol{I}}$ passes through at least three growing points(include $\boldsymbol{P}_{\boldsymbol{l}}$ and $\boldsymbol{P}_{2}$ ) while no point in $\boldsymbol{S}_{\boldsymbol{V}}$ inside it. So the distance between $\boldsymbol{P}$ and each of the three growing points equals to the distance between $\boldsymbol{P}$ and each of other two growing points, and is equal or lesser than the distance between $\boldsymbol{P}$ and each of other growing points in $\boldsymbol{S}_{\boldsymbol{V}}$. And so point $\boldsymbol{P}$ is the public vertex of the Voronoi polygon correspondence with the three growing points. In the same way, point $\boldsymbol{Q}$ is also the public vertex of the Voronoi polygon correspondence with the corresponding three growing points of $\boldsymbol{Q}$ (include $\boldsymbol{P}_{\boldsymbol{1}}$ and $\boldsymbol{P}_{2}$ ). Then, that an arbitrary point $\boldsymbol{M}$ on segment $\boldsymbol{P} \boldsymbol{Q}$ must be on the public edge of the Voronoi polygon correspondence with $\boldsymbol{P}_{\boldsymbol{1}}$ and $\boldsymbol{P}_{2}$, can be proved. This is because, for
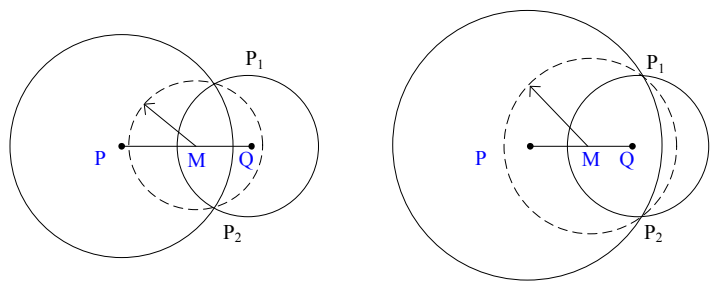

Fig. 2. Two cases of intersecting circles 
circle $\boldsymbol{O}_{\boldsymbol{M}}$ centered at $\boldsymbol{M}$ and passing through $\boldsymbol{P}_{\boldsymbol{1}}$ and $\boldsymbol{P}_{2}, O_{M}<O_{1} \cup O_{2}$, and so the distance between $\boldsymbol{M}$ and $\boldsymbol{P}_{\boldsymbol{1}}$ equals to the distance between $\boldsymbol{M}$ and $\boldsymbol{P}_{2}$, and is equal or lesser than the distance between $\boldsymbol{M}$ and each of other growing points in $\boldsymbol{S}_{\boldsymbol{V}}$. Consequently, segment $\boldsymbol{P Q}$ is the public edge of the Voronoi polygon correspondence with $\boldsymbol{P}_{\boldsymbol{1}}$ and $\boldsymbol{P}_{\boldsymbol{2}}$. On the basis of the concept of Voronoi diagram, the necessity of theorem 2 is easy to be proved, and is omitted here.

\section{Algorithm of 2D Conforming Voronoi Diagram Generation in Complex Domain}

Definition 2. Given a PSLG $\boldsymbol{A}$, the local feature size Ifs $(\boldsymbol{p})$ of a point $\boldsymbol{p}$ with respect to $\boldsymbol{A}$ is the minimum distance between $\boldsymbol{p}$ and the elements of $\boldsymbol{A}$ that do not contain $\boldsymbol{p}$. Obviously, $\operatorname{lfs}(p)>0$.

As shown in Figure 3, the size of the circle represents the local feature size of corresponding point.

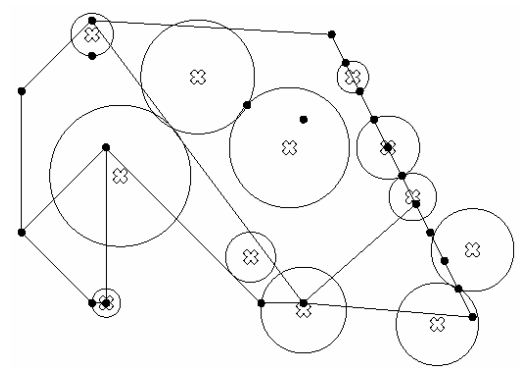

Fig. 3. Local feature size

Here is the description for generation algorithm of 2D conforming Voronoi diagram in complex domain.

Algorithm generation algorithm of 2D conforming Voronoi diagram in complex domain

Input PSLG $A(A=P S \bigcup S S)$

Output conforming Voronoi diagram which fulfills PSLG $\boldsymbol{A}$

Step1: For each endpoint $\boldsymbol{P}$ of each segment of $\boldsymbol{S S}$, construct a circle with center $\boldsymbol{P}$ and radius $\alpha \cdot \operatorname{lfs}(P)(0<\alpha<1 / 2$, typically $\alpha=0.45)$.

Step2: For $\forall p \in P S$, add $\boldsymbol{p}$ to growing point set $\boldsymbol{S}_{\boldsymbol{V}}$.

Step3: For $\forall P Q \in S S$, all the circles with center on segment $\boldsymbol{P Q}$ constructed during algorithm process are denoted by $\operatorname{CS}(\boldsymbol{P} Q)$. Obviously, at first $\operatorname{CS}(\boldsymbol{P Q})$ only contains two endpoint circles with radius $\alpha \cdot \operatorname{lfs}(P)$ and $\alpha \cdot \operatorname{lfs}(Q)$ respectively. Because $\alpha \cdot \operatorname{lfs}(P)+\alpha \cdot \operatorname{lfs}(Q)<|P Q|$, there is sub-segment of $\boldsymbol{P Q}$ not covered by 
circles in set $\operatorname{CS}(\boldsymbol{P Q})$, and the endpoints of sub-segment are the intersection points of $\boldsymbol{P} \boldsymbol{Q}$ with the circles in $\operatorname{CS}(\boldsymbol{P} \boldsymbol{Q})$. All the sub-segments of $\boldsymbol{P} \boldsymbol{Q}$ not covered by circles in set $\operatorname{CS}(\boldsymbol{P Q})$ are denoted by $\operatorname{US}(\boldsymbol{P} \boldsymbol{Q})$.

For $\forall P Q \in S S$, following operation is performed: $\forall b_{1} b_{2} \in \operatorname{US}(P Q)\left(\boldsymbol{b}_{1}\right.$ and $\boldsymbol{b}_{2}$ are the intersection points of $\boldsymbol{P Q}$ with circle $\boldsymbol{C}_{\boldsymbol{1}}$ and $\boldsymbol{C}_{2}$ in $\operatorname{CS}(\boldsymbol{P Q})$ respectively, the centers of $\boldsymbol{C}_{\boldsymbol{1}}$ and $\boldsymbol{C}_{2}$ are $\boldsymbol{O}_{\boldsymbol{1}}$ and $\boldsymbol{O}_{2}$ respectively, and the radiuses of $\boldsymbol{C}_{\boldsymbol{1}}$ and $\boldsymbol{C}_{2}$ are $\boldsymbol{r}_{\boldsymbol{1}}$ and $r_{2}$ respectively.), construct a new circle with radius $r=\min \left(\alpha \cdot \operatorname{lfs}(O),\left|O b_{1}\right|+r_{1} / 2,\left|O b_{2}\right|+r_{2} / 2\right)$ at point $\boldsymbol{O}$ on segment $\boldsymbol{b}_{1} \boldsymbol{b}_{2}$, and add the new circle to $\operatorname{CS}(\boldsymbol{P Q})$. If $0 \leq\left(r-\left|O b_{1}\right|\right) / \min \left(r, r_{1}, r_{2}\right)<\beta(0<\beta<1 / 2$, typically $\beta=0.1)$, let $r=(1-\beta) \cdot r$ to make the new added circle not intersect circle $\boldsymbol{C}_{\boldsymbol{I}}$ and $\boldsymbol{C}_{2}$. Repeat the operation above till US(PQ) becomes empty.

Step4: For $\forall P Q \in S S$, calculate the intersection points of two adjacent circle in $\mathrm{CS}(\boldsymbol{P Q})$, and add calculated intersection points to growing point set $\boldsymbol{S}_{\boldsymbol{V}}$.

Step5: If vertex $\boldsymbol{P}$ in PSLG $\boldsymbol{A}$ is one endpoint of just only one segment $\boldsymbol{P} \boldsymbol{Q}$, let backward extension line of $\boldsymbol{P Q}$ intersect circle $\boldsymbol{C}$ centered at $\boldsymbol{P}$, and add the intersection point to growing point set $S_{V}$.

Step6: Generate Delaunay triangulation of growing point set $S_{V}$ first, then compute the Voronoi polygon for each growing point in $S_{V}$ by duality: for each growing point $p \in S_{V}$ internal to the underlying domain, traverse the triangles pertaining to $\boldsymbol{p}$ counterclockwise and connect the corresponding nodes in order. In this step, if optimized Delaunay triangulation generation algorithm is used, operation complexity would be $O(N \log N)$.

Union of computed Voronoi polygons above is right conforming Voronoi diagram fulfilling PSLG $\boldsymbol{A}$.

\section{Algorithm Convergence Analysis}

Whether the algorithm proposed above converges depends on whether Step3 converges, namely for $\forall P Q \in S S$, whether there exists a finite set $\operatorname{CS}(\boldsymbol{P Q})$ which would cover segment $\boldsymbol{P} \boldsymbol{Q}$. To prove that algorithm is convergent, give the following lemma first.

Lemma 1. For $\forall P Q \in S S$, local feature size function lfs is continuous on the open segment $\boldsymbol{P Q}$.

Here is proof for algorithm convergence.

Proof. For $\forall P Q \in S S$, when Step1 has just been performed, there exists only two endpoint circles with radiuses $\alpha \cdot \operatorname{lfs}(P)$ and $\alpha \cdot \operatorname{lfs}(Q)$ respectively in $\operatorname{CS}(P Q)$. At the moment, there exists only one sub-segment $\boldsymbol{P}_{\boldsymbol{l}} \boldsymbol{Q}_{\boldsymbol{l}}$ in $\operatorname{US}(\boldsymbol{P Q})$, and endpoint $\boldsymbol{P}_{\boldsymbol{l}}$ and $\boldsymbol{Q}_{\boldsymbol{1}}$ of $\boldsymbol{P}_{1} \boldsymbol{Q}_{1}$ are the intersection points of $\boldsymbol{P Q}$ with the two endpoint circles respectively. By Lemma1, lfs is continuous on close segment $\boldsymbol{P}_{\boldsymbol{l}} \boldsymbol{Q}_{\boldsymbol{l}}$, further more, Ifs is bounded on $\boldsymbol{P}_{1} \boldsymbol{Q}_{1}$ as inferred from the property of continuous function on bounded 
closed region. Hence, for lfs, there exists a minimum value $\mathrm{d}$ on close segment $\boldsymbol{P}_{\boldsymbol{l}} \boldsymbol{Q}_{\boldsymbol{l}}$, and obviously, $\mathrm{d}>0$. Then in Step3, when a new circle is added to $\operatorname{CS}(\boldsymbol{P Q})$, it will result in one of the following two cases: (1)It will decrease the number of subsegments in US(PQ) by one and decrease the total length of sub-segments in $\operatorname{US}(\boldsymbol{P Q})$. (2)It will increase the number of sub-segments in $\operatorname{US}(\boldsymbol{P Q})$ by one and decrease the total length of sub-segments in $\operatorname{US}(\boldsymbol{P Q})$ by at least $2 \cdot \alpha \cdot(1-\beta) \cdot \mathrm{d}$. Obviously, both the two cases can happen only a finite number of times. Consequently, after adding finite circles to $\operatorname{CS}(\boldsymbol{P Q})$, the number of sub-segments in $\operatorname{US}(\boldsymbol{P Q})$ will become zero, namely, there exists a finite set $\operatorname{CS}(\boldsymbol{P Q})$ which would cover segment $\boldsymbol{P Q}$.

\section{Examples}

The experiment was performed using Visual C++ and OpenGL. Hardware Environment mainly consists of CPU Core2 Duo T6670 2.2GHz, Memory 2G, Video Memory 512M, and Operating System is Windows XP SP2.

Conforming conditions include conforming point (well point) and conforming line (outer boundary and inner fault line).

Take PSLG $\boldsymbol{A}$ showed in Figure 4(a) as input and generate conforming Voronoi diagram showed in Figure 4(b) using the algorithm proposed in the paper, which takes 1867 milliseconds to generate the 2D conforming Voronoi diagram. The PSLG B shown in Figure 5 takes 7831 milliseconds to generate the conforming Voronoi

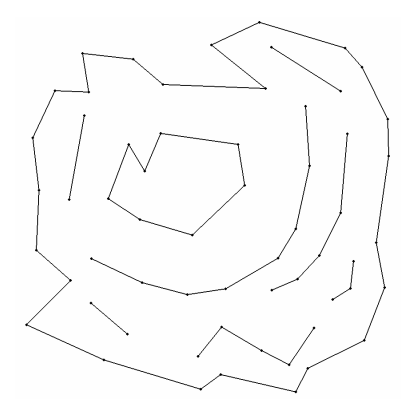

(a) PSLG $A$

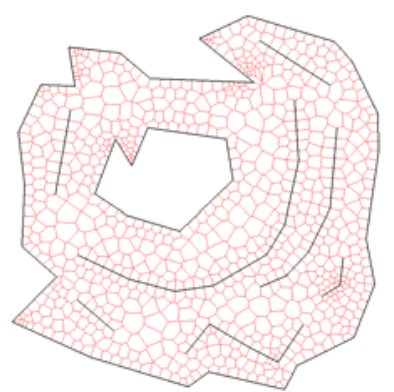

(b) Generated Voronoi diagram

Fig. 4.

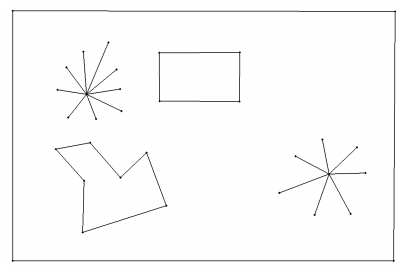

(a) PSLG $B$

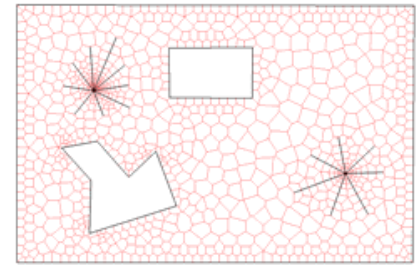

(b) Generated Voronoi diagram

Fig. 5. 
diagram. Experiments indicate that the algorithm proposed in the paper can effectively deal with inner boundary, linear conforming conditions and irregular area to generate conforming Voronoi diagram with good quality.

\section{Conclusion and Future Work}

A generation algorithm of $2 \mathrm{D}$ conforming Voronoi diagram in complex domain is proposed in this paper. Besides oil reservoir numerical simulation field, the algorithm proposed in this paper can also fulfill requirements of many other fields, such as fluid mechanics, biology, geostatistical analysis and so on. Future work are researching on $3 \mathrm{D}$ conforming Voronoi diagram generation in complex domain.

Acknowledgment. This research is partially supported by Important National Science \& Technology Specific Projects 2008ZX05038-001 and Funding Project for Academic Human Resources Development in Institutions of Higher Learning Under the Jurisdiction of Beijing Municipality PHR201008239.

\section{References}

1. Heinemann, Z.E., Brand, C.W.: Gridding techniques in reservoir simulation. Presented at First and Second International Forum on Reservoir Simulation, pp. 339-426 (1989)

2. Melichar Herber, T., Reingruber, A.J., Shotts, D.R., et al.: Use of PEBI grids for a heavily faulted reservoir in the Gulf of Mexico. In: Proceedings of SPE Annual Technical Conference and Exhibition, pp. 2755-2764. Denver (2003)

3. Xie, H.: Research on unstructured grid oil reservoir numerical simulation method. Institute of Petroleum Exploration and Design, Beijing (1999)

4. Xie, H., Heng, G., Guo, S., Yin, D.: PEBI grid 2D two-phase flow numerical simulation. Acta Petrolei Sinica 20(2), 57-61 (1999)

5. Verma, S.: A flexible gridding scheme for reservoir simulation. Presented at SPE Intl, Student Paper Contest, pp. 657-672 (1995)

6. Kappa.: Saphir V 3.0 technical reference. Engineering Company, Paris (2000)

7. Xiang, Z., Zhang, L., Chen, Z.: Generation algorithm of oil reservoir arbitrary conforming plane domain PEBI grid. Journal of Southwest Petroleum Institute 28(2), 32-35 (2006)

8. Yang, Q., Cai, Q., Li, J.: Research on 2-D constained PEBI grid generation technique. Computer Engineering and Applications 22(7), 80-83 (2004)

9. Cai, Q., Yang, Q., Meng, X.: Research on 2D PEBI grid generation. Journal of Engineering Graphics 26(2), 69-72 (2005)

10. Li, J.: Conforming Voronoi generation. Beijing University of Aeronautics and Astronautics, Beijing (2006)

11. Yang, Q., Zhang, J., Li, J., Jin, M.: 2D conforming Voronoi mesh generation by refinement algorithm. Journal of Computer-Aided Design \& Computer Graphics 18(10), 1547-1552 (2006)

12. Preparata, F.P., Shamos, M.I.: Computational Geometry: an introduction. Springer, New York (1985)

13. Li, H.: Research on theory and visualization application of Delaunay triangulation. Harbin Institute of Technology Press, Harbin (2010) 